\title{
OPTIMAL REDUNDANCY OF RADIAL DISTRIBUTION NETWORKS BY CRITERIA OF RELIABILITY AND INFORMATION UNCERTAINTY
}

\author{
Dulesov Aleksandr Sergeevich \\ Karandeev Denis Jurevich \\ Katanov Khakass State University \\ Abakan, Russian Federation \\ den_dr_house_1991@mail.ru
}

\author{
Dulesova Natalia Valeriyvna \\ Khakass Technical Institute of Siberian Federal University \\ Abakan, Russian Federation \\ Dulesovanv@mail.ru
}

\begin{abstract}
The model is based on the criteria of costeffectiveness and information uncertainty. The created model has fundamental difference from the current classical economicmathematical models. As constraints it is proposed to use the mathematical expressions of the information entropy determination of two states of the system: operable and nonoperable. These expressions are the constraints imposed in the task that creates an optimal radial network with redundancy. Expressions are constructed as all the elements reserving the each other are similar, i.e. they have the same performance. The given example shows how to calculate the information entropy, where a constraint is a problem solution, as well as the possibility of both applications of probabilities and numerical values for the information entropy determination is proved.
\end{abstract}

Keywords - optimization, information uncertainty, information entropy, structural system reliability, network redundancy

\section{INTRODUCTION}

In practice of distribution network designing in order to increase the reliability level, we use the redundant of similar elements. At the same time, the redundancy account requires to increase cash flow that constrains the desire to achieve a high degree of object reliability. Therefore, it is necessary to distribute cash optimally while preserving the required network reliability [1].

Touching upon the problem how to find an optimal number of the redundancy elements, we are talking about two criteria: cost-efficiency and reliability [2]. The importance of these criteria is actually indisputable. However, the cost-efficiency account in the optimization problem is poorly formalized [3]. For example, the structural reliability is expressed through probabilistic feature of a network or through standard requirements where the account in optimization models is complicated. It is possible to overcome these difficulties, but according to some assumptions. The authors have published the list of papers [4-7], where the evaluation of the structural reliability of the technical system is possible through the calculation of information entropy $[8,9]$. Therefore, we are going to search the solution based on constructed optimization problem of the mathematical model, where cost-efficiency and information uncertainty criteria must be complied, which reflect a degree of distribution network reliability.

\section{THE CLASSICAL PROBLEM OF REDUNDANCY}

We assume that the original structure of the designed network has a radial form without redundancy. Each element $i$ of the model is characterized by indicators: construction costs $C_{i}$; the availability coefficient or probability of operable state (non-failure operation) $p_{i}$; the unavailability coefficient or probability of non-operable state (failure) $q_{i}$.

To determine indicators the failure statistics or normative values are used [10]. The probability of operable state is $p=r /(m+r)=r / T$ and non-operable state is $q=1-p$. Where $r$ - the mean time to recovery; $m=1 / \lambda$ - time between failures; $m+r=T$ - mean time between failures; 1 - parameter failure rate.

In reliability theory [11] the following tasks of optimal redundancy have been formulated:

1. Direct task: to find the $x^{*}$ vector that is the solution

$$
\min \left\{p(x): C(x) \geq C^{0}\right\} \text { or } \min \left\{q(x): C(x) \leq C^{0}\right\},
$$

where $p(x)$ and $q(x)$ - minimized probabilities; $C(x)$ reserve costs; $C^{0}$ - given resource constraints

2. The inverse task: to find the $x^{*}$ vector that is the solution

$$
\min \left\{C(x): p(x) \geq p^{0}\right\} \text { or } \min \left\{C(x): q(x) \leq q^{0}\right\} .
$$

In (1) and (2) tasks the reliability criterion of the scheme is expressed through the state probabilities of the elements that allow us to apply methods of the information entropy 
determination [12]. They are based on the K. Shannon principle of "the choice of a variety of alternatives" [13].

\section{MODEL OF PARALLEL REDUNDANT NETWORK}

The classical determination of the Shannon information [14] implies the existence of statistical group: time (an element has been in a particular state); frequency of occurrence of certain events, etc. [15]. These indicators of structural reliability allow us to calculate the probability of finding the element $i$ in two opposite states: the probability of operable $p_{i}$ and non-operable $-q_{i}=1-p_{i}$ states. As we consider only two states, we can determine the information (statistical) entropy by Shannon for a single element $i$ according to the expression:

$$
H_{i}=-\left(p_{i} \log _{2} p_{i}+q_{i} \log _{2} q_{i}\right) \text {, if } p_{i}+q_{i}=1 \text {. }
$$

Here the determination logarithm of value $H_{i}$ in bits has a base 2, which indicates two opposite states: operable state (non-failure operation) and non-operable state (failure).

If we consider the radial network as a set of elements excluding the connections between them (in case of independent functioning of the elements) then it is easily possible to determine the total entropy (the amount of information uncertainty) of all the elements:

$$
\begin{aligned}
& H_{\Sigma}=-\sum_{i=1}^{N}\left[H\left(p_{i}\right)+H\left(q_{i}\right)\right]= \\
& =-\sum_{i=1}^{N}\left(p_{i} \log _{2} p_{i}+q_{i} \log _{2} q_{i}\right),
\end{aligned}
$$

where $H\left(p_{i}\right)$ and $H\left(q_{i}\right)$ - the entropy of the operable and non-operable states of the network elements, $N$ - number of the elements in the network structure.

The entropy maximum $H_{\Sigma}=N$ according to (4) is achievable when all $p_{i}=q_{i}=0,5$ are equal. For the network the value $H_{\Sigma}$, obtained according to (4) will remain invariable, as in this case situations are not associated with the arrival or disposal of information according to the given network. For example, if any element was not included in the network or not excluded from the network. Then for a real network with elements connections the obtained values for the information entropy of the operable $H(P)$ and non-operable $H(Q)$ states of the elements must consider the probable joint state of elements according to $[16,17]$.

The solution of problems (1) and (2) comes to the construction of a parallel network structure where joint elements form redundancy groups (Fig. 1).

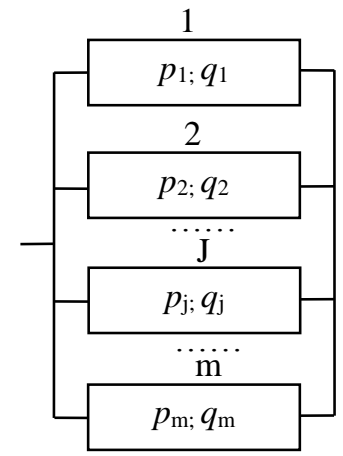

Figure 1 - Parallel network structure

According to [18], the expression of total entropy determination for serial-parallel structures based on the entropy differentiation into components for two states operable (non-failure operation) and non-operable (failure) and $m$ elements in a redundant group $j$ can be written as:

- for non-operable state of group elements:

$$
H\left(Q_{j}\right)=-\sum_{i=1}^{m}\left[\left(\prod_{\substack{k=1 \\ k \neq i}}^{m} q_{k}\right) H\left(q_{i}\right)\right]
$$

- for operable state of group elements:

$$
H\left(P_{j}\right)=-\sum_{k=1}^{m}\left[H\left(p_{k}\right)+H\left(q_{k}\right)\right]+H\left(Q_{j}\right)=H_{\Sigma}-H\left(Q_{j}\right)
$$

According to (5) and (6) $k$ - an ordinal number of the element in a group $j, m-$ amount of redundant elements in the group.

\section{THE MATHEMATICAL MODEL OF REDUNDANCY RADIAL NETWORK}

Further, we are going to present an optimization problem (2) with the measure of information uncertainty where $\min \left\{C(x): H(P) \geq H^{0}(P)\right\}$ or $\min \left\{C(x): H(Q) \leq H^{0}(Q)\right\}$ where $H^{0}(P)$ and $H^{0}(Q)$ - boundary (critical) entropy value, that sometimes can fail a required number of redundant elements. Let's put forward a condition: all redundant elements in a group have identical values of indicators, i.e. $p_{1}$ $=p_{2}=\ldots=p_{k}=\ldots=p_{m}$ and $q_{1}=q_{2}=\ldots=q_{k}=\ldots=q_{m}$. Let's indicate the size of $j$ through $x_{j}$ a redundancy group (it is a total number of parallel connected of the similar redundancy elements). Then the entropy of non-operable state of the group is:

$$
H\left(Q_{j}\right)=-x q^{x-1} H(q)=-x q^{x} \log _{2} q .
$$

According to (7) and further the $j$ symbol at $x$ and $q$ is not specified in order to avoid the bulkiness formulas. And 
according to (6) and (7), the entropy of the operable state of the group is:

$$
\begin{aligned}
& H\left(P_{j}\right)=-x\left(p \log _{2} p+q \log _{2} q-q^{x} \log _{2} q\right)= \\
& =-x\left(H-q^{x} \log _{2} q\right)
\end{aligned}
$$

Keeping to the constraint condition on with a number of information entropy and using (8) let's write the constraint equations:

$$
\text { - for } H^{0}(P): H-q^{x} \log _{2} q \geq H^{0}(P) \text {; }
$$

$$
\text { - for } H^{0}(Q): q^{x} \log _{2} q \leq H^{0}(Q) \text {. }
$$

Then the optimization problem can be written as:

$$
\begin{aligned}
& C\left(x_{j}\right)=\sum_{j=1}^{n} c_{j} x_{j} \rightarrow \min ,
\end{aligned}
$$

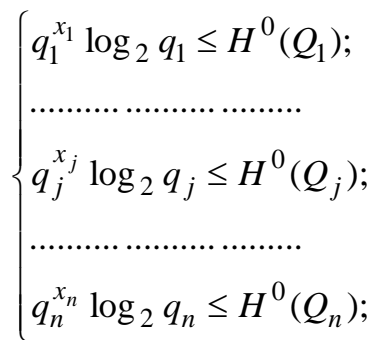

where $x_{j} \geq 0, j=1,2, \ldots, n, n-$ a number of groups in the radial network.

The system of constraints (12) can be converted to:

$$
\left\{\begin{array}{l}
a_{1} q_{1}^{x_{1}} \leq H^{0}\left(Q_{1}\right) \\
\ldots \ldots \ldots \ldots \ldots \ldots \ldots \ldots \ldots \ldots \ldots \ldots \ldots \\
a_{j} q_{j}^{x_{j}} \leq H^{0}\left(Q_{j}\right) \\
\ldots \ldots \ldots \ldots \ldots \ldots \ldots \ldots \\
a_{n} q_{n}^{x_{n}} \leq H^{0}\left(Q_{n}\right)
\end{array}\right.
$$

where $-a_{j}=\log _{2} q_{j}$ the coefficient of a nonlinear equation.

When solving the (11) - (12) system it is necessary to determine the boundary value of entropy $-H^{0}\left(Q_{j}\right)$. And its role and importance should be clear to the designer. To calculate the $H^{0}\left(Q_{j}\right)$ value in (12) or (13), it is possible to represent it as a percentage ratio with the total entropy of an element of a redundancy group $j$ :

$$
H_{\%}^{0}\left(Q_{j}\right)=\frac{H\left(Q_{j}\right)}{H_{j}} \cdot 100,
$$

The obtained value allows to determine $H_{\%}^{0}\left(P_{j}\right)=100-H_{\%}^{0}\left(Q_{j}\right)$.

Further, on the example we are going to show how to determine $H^{0}\left(Q_{j}\right)$.

Let's assume:

a) the redundancy is absent $(x=1), p=0,99$ is (for high reliable components) and $q=1,0-0,99=0,01$ then according to $(14)-H_{\%}^{0}\left(Q_{j}\right)=82,23 \%, H_{\%}^{0}\left(P_{j}\right)=17,77 \%$;

b) the redundancy consists of 2 elements $(x=2)$ and $p=0,99$ $-H_{\%}^{0}\left(Q_{j}\right)=0,82 \%$;

c) the redundancy consists of 3 elements $(x=3)$ and $p=0,99$ - $H_{\%}^{0}\left(Q_{j}\right)=0,01 \%$. Having a boundary value in percentage according to (14) it is possible to determine the boundary value of entropy in bits, that is necessary for its substitution in the constraint system of the optimization problem.

The obtained values (in different options of redundancy) don't reflect the real ratio of the boundary values of the entropy with the given probability values. The matter is that for the actual network with the highest reliable elements, the information entropy of its non-failure operation is $H_{\%}^{0}\left(P_{j}\right)>>H_{\%}^{0}\left(Q_{j}\right)$. However, for variant a) this condition isn't fulfilled. In order that this condition has been met, it is possible to replace probabilities in the logarithm with the values greater than 1,0 .

\section{THE AVERAGE TIME OF STATES}

Let's consider the application as parameters, according to [19], the average time of operable $M_{p i}$ and non-operable $M_{q i}$ states of an element $i$ and using these values we can find the probabilities $p_{i}=M_{p i} / T$ and $q_{i}=M_{q i} / T$, where $T=M_{p i}+M_{q i}$ is a number of hours in a year. The value of information entropy of an element will be determined by the expression:

$$
\begin{aligned}
& H_{i}=p_{i} \log _{2} M_{p i}+q_{i} \log _{2} M_{q i} ; \\
& H\left(Q_{j}\right)=q^{x} \log _{2} M_{q i} ; \text { by } p_{i}+q_{i}=1 .
\end{aligned}
$$

The problem solution of (11) - (12) allows to use $M_{p i}$ and $M_{q i}$, thus to determine $H_{\%}^{0}\left(Q_{j}\right)$. Let's calculate the given entropy in the previous example (variant a) after having set $T=10000$ hours: redundancy is absent $(x=1)$; mean time between failures $(\mathrm{MTBF})-M_{p i}=9900$ hours (corresponding to 
$p=0,99)$; mean time to restoration (MTTR) $-M_{q i}=100$ hours $(q=0,01)$. Then, according to (14) and $(15)-H\left(Q_{j}\right)=0,066$, $H_{i}=13,207, \quad H_{\%}^{0}\left(Q_{j}\right)=0,05 \%, \quad H_{\%}^{0}\left(P_{j}\right)=99,5 \%$. The obtained values confirm the adequacy of the boundary evaluation with the redundancy conditions.

To calculate the $H^{0}\left(Q_{j}\right)$ value it is sufficient to set the expected number of non-operable hours $M_{q j}$ of group state and to determine it with the help of (14) and (15). For example, the average time of an element emergency outage or the energy consumer $M_{q}$ must not be less than 175 hours per year. Then mean time between failures (MTBF) (based on 10,000 hours of the system operation) is $M_{p}=9825$ hours. According to the probability: $p=0.9825 ; q=0.0175$. The critical value of information entropy for a group of elements: $H=H\left(M_{p}\right)+H\left(M_{q}\right)=p \log _{2} M_{p}+q \log _{2} M_{q}=$ $=0,9825 \log _{2} 9825+0.0175 \log _{2} 175=13.161$.

$H_{\%}^{0}\left(Q_{j}\right)=\frac{H\left(M_{q}\right)}{H} \cdot 100=\frac{0.13}{13.161} \cdot 100=0.991 \%$ or $H^{0}\left(Q_{j}\right)=\frac{H\left(M_{q}\right)}{H}=\frac{0.13}{13.161}=0.0099$. Thus, the boundary value of information entropy (based on the above conditions is $M_{q}=175$ hours) for the group of elements $j$ we take: $H^{0}\left(Q_{j}\right)=0.01$.

The problem solution of $(11)-(13)$ is possible with the application of classical methods of nonlinear programming [20].

\section{CONCLUSION}

The task construction of the optimal structure of the radial distribution networks with redundant elements assumes the creation of economic and mathematical model meeting at least two criteria. The first is well-known economic one; the second is the information uncertainty that reflects the level of the network structural reliability. And it is the last criterion that is taken as a constraint to search the problem solutions. The constructed constraints as nonlinear equations of the information entropy determination form a feasible region of the problem. In fact, a feasible region is a set of all possible solutions where an obtained number of redundancy elements will carry the information that the network operation reliability meets the requirements imposed by designers and operators.

\section{ACKNOWLEDGMENT}

The research has been performed with support from the Russian Foundation for Basic Research (project no. 15-0801473a).

\section{REFERENCES}

[1] Sankaraiah G., Raghunatha Reddy Y. "Design and optimization of an Integrated Reliability redundancy system with multiple constraints"
2010 2nd International Conference on Reliability, Safety and Hazard Risk-Based Technologies and Physics-of-Failure Methods (ICRESH), Mumbai, 2010, pp. 118-122. doi: 10.1109/ICRESH.2010.5779527

[2] Dulesov A.S., Karandeev D. Y. Construction of optimal structure of technical system using "branch and bound" by criteria of profitability and reliability // "Journal Safety \& reliability of Power Industry". - 2016 - vol. 33, 56-59. (in Russian, abstr. in English).

[3] Farag Reda, Achintya Haldar, A novel reliability evaluation method for large engineering systems // Ain Shams Engineering Journal, February 2016, Pages 1-13.

[4] Dulesov A.S., Kondrat N.N. Measure of indefiniteness of information and its properties in relation to the assessment of random behavior of a technical object // Journal "Science Review". - 2014 - vol. 7, 258-264. (in Russian, abstr. in English).

[5] Dulesov A.S., Kondrat N.N. Amount equivalenting of information entropy in the technical system structure // Journal "Fundamental research". - 2015 - vol. 6, pt. I., 14-19. (in Russian, abstr. in English).

[6] Dulesov A.S., Karandeev D. Y., Kondrat N.N. How to define amount of information for the simplest structure of system // Vestnik of Katanov Khakass State University. - 2016 - vol. 15, 7-9. (in Russian, abstr. in English).

[7] Dulesov A.S., Karandeev D. Y., Kondrat N.N. Definition of amount of information entropy in structure of the technical system by method of the minimum ways // Journal "Modern high technologies". - 2016 - vol. 2, pt. III., 425-429. (in Russian, abstr. in English).

[8] Hartley, R.V.L., "Transmission of Information”, Bell System Technical Journal, Volume 7, Number 3, pp. 535-563, (July 1928).

[9] Kolmogorov A. Three approaches to the quantitative definition of information // International Journal of Computer Mathematics, 1965. T.1, №1 - C. 3-11.

[10] Ebeling, Charles E., (1997), An Introduction to Reliability and Maintainability Engineering, McGraw-Hill Companies, Inc., Boston.

[11] Ushakov, I. A. "Solving of optimal redundancy problem by means of a generalized generating function". Journal of Information Processing and Cybernetics archive. Vol. 24 Issue 4-5, 1988 pp. $219-222$.

[12] Dulesov A.S., Ageeva P.A. Information measure in the problem of the estimation of trouble-free work of technical system // Journal "Fundamental research". - 2011 - vol. 12, pt. I., 102-107. (in Russian, abstr. in English).

[13] Shannon, C.E. "Mathematical Theory of Communication". Bell System Tech. J., 1948, no. 27, pt. I., 379-423; pt. II., 623-656.

[14] Cover, Thomas M. and Joy A. Thomas. Elements of Information Theory, second edition, 2006. New Jersey: Wiley and Sons.

[15] Shannon, C.E. "Communication Theory of Secrecy Systems",. Bell System Tech. J., vol. 28, pp. 656-715, Oct., 1949.

[16] Dulesov A.S., Kondrat N.N. Measure of indefiniteness of information and its properties in relation to the assessment of random behavior of a technical object // Journal "Science Review”. - 2014. №7(1). 258-264.

[17] Dulesov A.S., Dulesova N.V., Karandeev D. Y. Delimitation indicator of level of reliability of technical system on the qualitative character: entropy approach // Journal "Fundamental research". - 2016 - vol. 2, pt. III., 477-481. (in Russian, abstr. in English).

[18] Dulesov A.S., Karandeev D. Y., Kondrat N.N. Definition of amount of information entropy in structure of the technical system by method of the minimum sections // Journal "Fundamental research". - 2016 - vol. 2, pt. III., 425-429. (in Russian, abstr. in English).

[19] Dulesov A.S., Karandeev D. Y., Kondrat N.N. Possibilities of application of expression of Shannon when determining amount of information // Vestnik of Katanov Khakass State University. - 2016 vol. 15, 5-7. (in Russian, abstr. in English).

[20] Luenberger, David G.; Ye, Yinyu (2008). Linear and nonlinear programming. International Series in Operations Research \& Management Science. 116 (Third ed.). New York: Springer. pp. xiv +546 . 